\title{
THE ROLE OF SATURN'S OBLATENESS IN THE MIMAS-TETHYS RESONANCE
}

\author{
R. GREENBERG \\ Lunar and Planetary Laboratory, University of Arizona, Tucson, Ariz. 85721, U.S.A.
}

Saturn's satellites Mimas and Tethys appear to be involved in a unique resonance. All orbit-orbit resonances, by definition, have the satellites' conjunction librating about some specific longitude. Equivalently, their orbital periods, measured relative to that longitude, are commensurable. Most orbit-orbit resonances are of the eccentricity-type; the conjunctions librate about the longitude of an apse of one orbit. The Mimas-Tethys resonance is of the inclination type.

These satellites have mean motions in a ratio of nearly $2: 1$. They have significant inclination and nearly circular orbits. Their masses are comparable so that their mutual perturbations are important. The masses contrast with, for example, the Titan-Hyperion resonance where the effect of Hyperion on Titan is negligible. The Mimas-Tethys conjunction librates about the midpoint of the longitudes of the satellites' nodes on Saturn's equatorial plane. This configuration holds true even as the nodes precess at rates on the order of a revolution per year (Brouwer and Clemence, 1961).

While the thrust of recent research has been toward explaining the origin of resonances, it is worthwhile to examine the physical mechanism which currently maintains this unique Mimas-Tethys case. The object is to explain this resonance in a physical way; the mathematical analysis has already been extensive and quite successful in describing the properties of this satellite interaction (e.g. Allan, 1969).

The typical mathematical analysis of this resonance is a study of the behavior of the resonance variable

$$
\Psi \equiv 4 \lambda_{\mathrm{T}}-2 \lambda_{\mathrm{M}}-\Omega_{\mathrm{T}}-\Omega_{\mathrm{M}},
$$

where $\lambda$ is the mean longitude and $\Omega$ is the longitude of the ascending nodes. The longitude of conjunction of the two satellites is given by $\xi \equiv 2 \lambda_{\mathrm{T}}-\lambda_{\mathrm{M}}$ and the average longitude of the ascending nodes is $\Omega_{\mathrm{AVG}} \equiv\left(\Omega_{\mathrm{T}}+\Omega_{\mathrm{M}}\right) / 2$. Clearly the resonance variable can be interpreted as twice the difference between the longitude of conjunction and the average longitude of the nodes. The second derivative is evaluated by Lagrange's planetary equations, ignoring short-period terms in the disturbing function and ignoring high orders of small quantities, leaving

$$
\ddot{\Psi}=-C I_{\mathrm{M}} I_{\mathrm{T}} \sin \Psi,
$$

where the quantity $C$ is positive. Thus $\Psi$ is stable at 0 , i.e. conjunction is stable at the average of the nodes.

As outlined here, the analysis appears to hold for any reference plane from which the inclinations are small, not only for the equatorial plane. But changing the reference 
plane slightly can drastically alter $\Omega_{\mathrm{AVG}}$. Why does the Mimas-Tethys conjunction librate about $\Omega_{\mathrm{AVG}}$ measured on the equatorial plane instead of on some other reference plane? The answer to this question is that from the point of view of the satellites' dynamics, the equatorial plane is special because of Saturn's oblateness.

In fact the oblateness is included implicitly in the analysis that I have described here. Some of the short-period terms that were neglected from the disturbing function contained

$$
\Psi_{1} \equiv 4 \lambda_{\mathrm{T}}-2 \lambda_{\mathrm{M}}-2 \Omega_{\mathrm{M}}=\Psi-\Omega_{\mathrm{M}}+\Omega_{\mathrm{T}}
$$

and

$$
\Psi_{2} \equiv 4 \lambda_{\mathrm{T}}-2 \lambda_{\mathrm{M}}-2 \Omega_{\mathrm{T}}=\Psi+\Omega_{\mathrm{M}}-\Omega_{\mathrm{T}}
$$

as arguments of cosines. These arguments have short periods compared to $\Psi$ only because of the rapid precession of $\Omega_{\mathrm{T}}$ and $\Omega_{\mathrm{M}}$ due to the oblateness of Saturn.

If we are to understand clearly the physical basis of this resonance we must be able to separate the role of the oblateness from the role of the mutual perturbations of the satellites. We can begin by first investigating the properties of inclination-type resonances with a model that does not include oblateness.

Consider the circular orbits of two satellites projected on a celestial sphere centered on their spherical primary (Figure 1). Let the north pole be defined by the total orbital angular momentum of the two satellites. Suppose the orbital mean motions are in a ratio of $2 / 1$ so that conjunction occurs repeatedly at the same meridian a few degrees after the satellite's mutual node as shown here. Since the mutual inclination is small, virtually no orbital energy is exchanged at conjunction by gravitational interaction.

However, energy is exchanged before and after conjunction. It is evident from Figure 1 that the gravitational effects are greater before conjunction because the

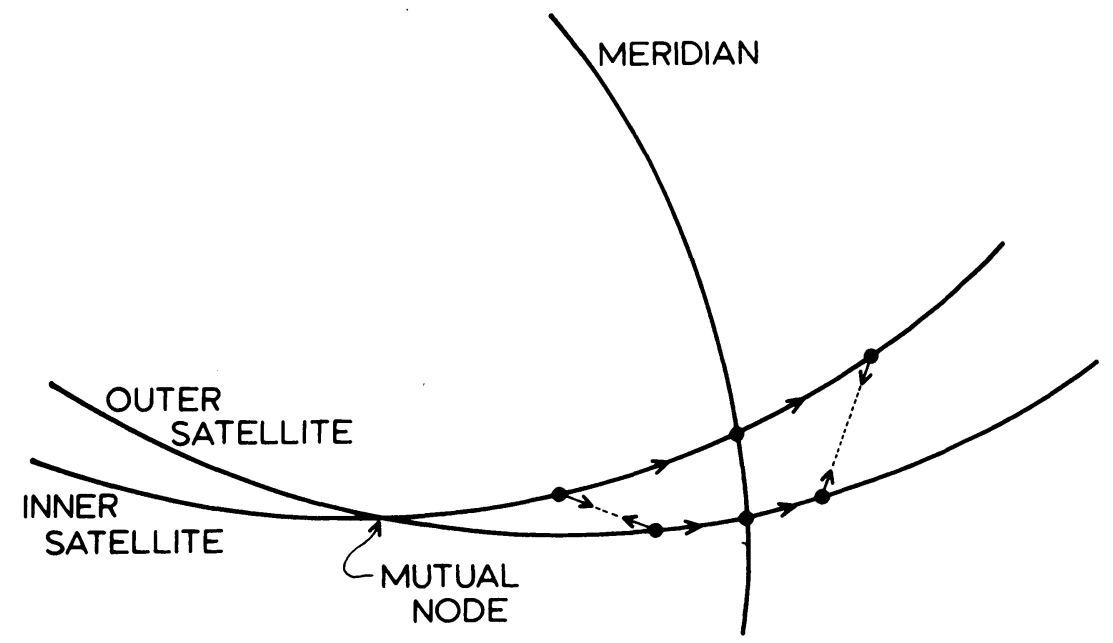

Fig. 1. 
satellites are closer to one-another than they are after conjunction; moreover, the lines of force are directed more nearly along the direction of motion before than after conjunction.

Thus the net effect of the gravitational interaction is to transfer energy from the outer satellite to the inner one as occurs before conjunction. This energy transfer slows the angular velocity of the inner satellite, lowering the mean motion ratio below the commensurable value $2 / 1$. Thus the next conjunction occurs further away from the mutual node of the satellites. Similarly if conjunction occurs before the mutual node, the net effect of the gravitational interaction is to cause conjunction to regress away from the mutual node.

Since there are two mutual nodes $180^{\circ}$ apart, conjunction is stable at either of the two longitudes $90^{\circ}$ from the satellites' mutual nodes. A more rigorous mathematical analysis has confirmed this result (Greenberg, 1973).

This type of resonance has two characteristics in common with eccentricity-type resonances. First, the stable condition is a 'mirror-configuration'. In other words, the velocities of the primary and its two satellites, measured relative to their center of mass, are normal to a plane containing all three masses. And second, conjunction is stable at that longitude where the orbits are furthest apart.

The inclination aspect of the Neptune-Pluto resonance is consistent with this description in that conjunction does librate about the longitude $90^{\circ}$ from the planets' mutual node (Williams and Benson, 1971). However, the Mimas-Tethys resonance does not appear to be consistent with this description. Let us consider what happens to our model if we make the primary oblate. The ascending node of each satellite on the equatorial plane of the primary now precesses due to the oblateness. But it can be shown that the mutual perturbations of the two satellites still tend to draw conjunction toward the longitudes $90^{\circ}$ from the mutual nodes. For want of a better name, we shall call these longitudes 'quasi-stable' since they are stable if the nodes do not precess.

We need to answer the following question: Why should conjunction librate about the midpoint of the nodes on the equatorial plane (as observed in the Mimas-Tethys case) instead of librating about the 'quasi-stable' longitude toward which it is drawn by mutual perturbations?

Let us compare the behavior of the various longitudes with time(Figure 2). Actually, the independent variable in Figure 2 is $\Delta \Omega$, the difference between the two ascending nodes on the equator. This quantity varies monotonically, and for our purposes, nearly linearly with time. For the Mimas-Tethys case, variation of $\Delta \Omega$ by $2 \pi$ takes place in about a year.

All longitudes plotted in Figure 2 are measured from the longitude of the node of the inner satellite. The dotted lines represent the mid-point of the longitudes of ascending nodes. This lon'gitude is a multi-valued function as shown. In this figure, the observed libration in the Mimas-Tethys case would be indicated by an oscillation of the conjunction longitude about a dotted line with a period of about $70 \mathrm{yr}$.

We also have plotted on this figure the quasi-stable longitudes. These have been 
calculated by straightforward spherical geometry and are shown as the solid wavy curves. These are the longitudes toward which the mutual gravitational effects tend to draw conjunction. The curves shown here were calculated for a case with the orbital inclinations equal to those in the Mimas-Tethys case. If these inclinations were equal,

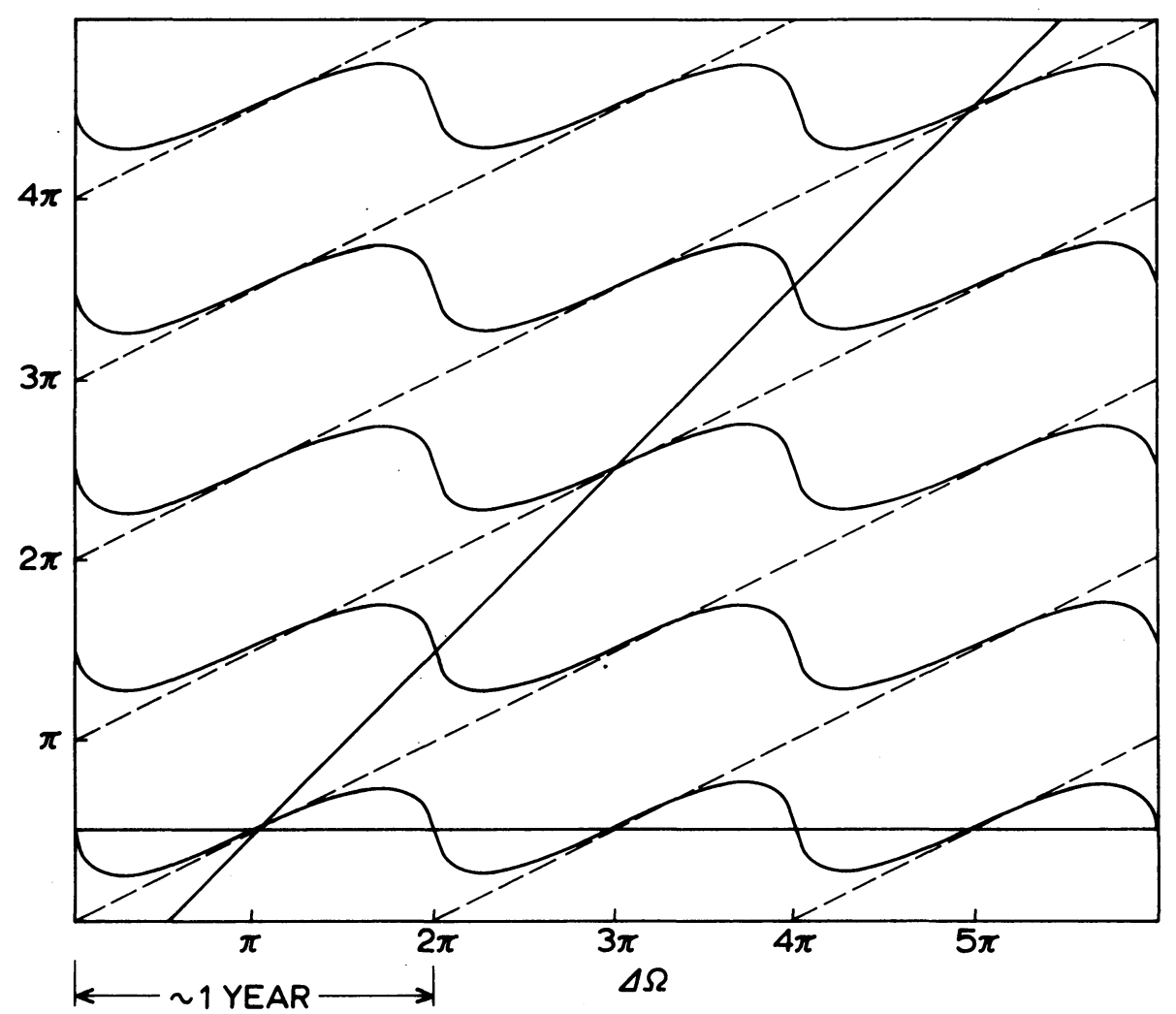

Fig. 2.

the curves would each be a sharp saw-tooth pattern with discontinuities at integer multiples of $2 \pi$. Our problem would be solved because in this case the quasi-stable longitudes would always be at the midpoint between equatorial nodes (Roy and Ovenden, 1955).

However, in the more realistic case where the inclinations are not equal, the curves of Figure 2 represent the quasi-stable longitudes. Suppose conjunction occurs at the midpoint between the equatorial nodes at an instant when the longitude is quasistable (e.g. at $\Delta \Omega=\pi$ ). At the quasi-stable longitude the mutual gravitational interaction does not accelerate conjunction one way or the other. Now suppose the ratio of the mean motions of the two satellites is such that the conjunction longitude follows one of the dotted lines. 
Immediately the mutual interaction tends to pull conjunction down toward the quasi-stable longitude. However, the time scale for response to this pull is quite long. (You will recall that the libration period for the Mimas-Tethys case is about $70 \mathrm{yr}$.) Before conjunction can respond significantly it has moved along the dotted line to a region where the mutual interaction tends to pull conjunction up toward the nearest quasi-stable longitude. As conjunction follows a dotted line, it experiences exactly as much pull up as down, so that the net effect is zero. In this sense, we see that conjunction at the midpoint of the equatorial nodes is an equilibrium configuration.

Is this a stable configuration? Suppose conjunction moves just below a dotted line. Most of the time it is pulled upwards toward the quasi-stable longitude so that the net effect over several years is to draw conjunction toward the dotted line. In this way we can show heuristically that conjunction is in stable equilibrium on the dotted line.

In summary, we have found that the instantaneous influence of mutual perturbation is to draw conjunction toward the quasi-stable longitudes, but that the average effect, due to the planet's oblateness, is to draw conjunction toward the observed stable longitudes.

I would like to point out that the mathematical theory of this resonance indicates that conjunction might also be stable at either satellite's descending node on Saturn's equator. Such longitudes can be represented in Figure 2 by the solid straight lines. Note that the net acceleration along either of these lines toward quasi-stable longitudes is zero, in perfect agreement with the mathematical theory.

In a sense this physical interpretation has not gone beyond the mathematical treatment in terms of revealing new properties of the Mimas-Tethys resonance. However, it is important to understand the mechanism maintaining the present resonance if we are to investigate successfully the origin of the resonance. In this regard, I would like to point out one more possible interpretation of this figure.

We can regard the solid curves as representing, at any instant in time, the positions of minima on a sinusoidal potential field governing the behavior of the conjunction longitude. As time goes on, the potential topography alternately follows the average nodes and then jumps back $180^{\circ}$ quite suddenly. In the case of the observed MimasTethys resonance, the sudden jumps occur too quickly for the system to respond. However, this effect may have played a role in the events that first locked these two satellites in resonance, when the amplitude of libration was nearly $180^{\circ}$. In this context, an understanding of the role of Saturn's oblateness is essential.

\section{References}

Allan, R. R.: 1969, Astron. J. 74, 497.

Brouwer, D. and Clemence, G. M.: 1961, in G. P. Kuiper and B. M. Middlehurst (eds.), Planets and Satellites, Univ. of Chicago Press, p. 31.

Greenberg, R. J.: 1973, Monthly Notices Roy. Astron. Soc. 165, 305.

Roy, A. E. and Ovenden, M. W.: 1955, Monthly Notices Roy. Astron. Soc. 115, 296.

Williams, J. G. and Benson, G. S.: 1971, Astwon. J. 76, 167. 


\section{DISCUSSION}

G. Hertz: How does the difference of the longitudes of the ascending nodes of Tethys and Mimas behave? And how is the effect of Titan?

$R$. Greenberg: To this approximation each longitude of the ascending node varies at a constant rate. Thus, their difference also varies with a constant rate. The discrepancy between this approximation and the true behavior of the difference is too small to affect the qualitative description of the resonance mechanism. Similarly, the effect of Titan would not change the basic mechanism which I have described. 